\title{
Tagungsforum
}

\section{Promovieren in der Rechtswissenschaft}

\author{
Arnd-Christian Kulow*
}

\begin{abstract}
A. „Verschulung“ der Promotion oder Forschung in „Einsamkeit und Freiheit"?
Das Zentrum für rechtswissenschaftliche Fachdidaktik (ZerF) an der Universität Hamburg beschäftigte sich auf seiner diesjährigen Tagung mit dem Thema der rechtswissenschaftlichen Promotion. Die Jahrestagung wurde gemeinsam mit der Albrecht Mendelssohn Bartholdy Graduate School am 18. und 19. März 2014 an der Universität Hamburg veranstaltet.
\end{abstract}

Promovieren und Rechtsdidaktik in einem Atemzug? Das kurze Zögern, das den Einen oder die Andere überkommen mag, führt mitten in die Thematik: Ist nicht die rechtswissenschaftliche Promotion das eigenständig anzufertigende Gesellenstück? Wo ist da Platz für „Didaktik“? Wie intensiv darf und sollte die Betreuung von Doktoranden also sein? Ist das Humboldtsche Ideal vom unabhängigen Wahrheitssucher obsolet?

Hochschulpolitik und Forschungsförderorganisationen sehen offenbar die Promotion zunehmend als weiteren schlichten Ausbildungsabschnitt und setzen daher verstärkt auf eine Formalisierung und generelle Institutionalisierung dieser Variante der Durchführung von Promotionsvorhaben. Vereinzelte, aber medial gleichwohl enorm breit diskutierte Fälle von Politikerdissertationen spielen dem scheinbar in die Hände. Das böse Wort von der „Statuspromotion" macht die Runde, und folgerichtig gerät dabei die Rechtswissenschaft ins Visier der Medien. Sie allein entscheidet über die Vergabe der Doktortitel. Sie muss daher auch über die zugrundeliegenden Kriterien Rechenschaft ablegen. Grund genug für das ZerF, diese Fragen in seiner diesjährigen Tagung zu thematisieren.

Tilman Repgen (Universität Hamburg) führte als derzeitiger Dekan und Mitglied des Direktoriums der Albrecht Mendelssohn Bartholdy Graduate School of Law aus einem historischen Blickwinkel in die Thematik ein. Im Mittelpunkt seines Referates stand der protestantische Theologe Paul Tillich, der - in den 30er-Jahren des vorigen Jahrhunderts - unter anderem das radikale Fragen zum Gegenstand seines Denkens gemacht hatte. Repgen relativierte damit gleich zu Beginn die Bedeutung der Frage nach der freien oder institutionalisierten Promotion. Mit Tillich machte er jedoch deutlich, worum es bei wissenschaftlichen Fragestellungen gehen sollte: um das radikale, d.h. an die Wurzeln gehende Fragen.

Über die „Qualität rechtswissenschaftlicher Promotionen: Rahmenbedingungen, Kriterien, Verfahren“ sprach Stephan Rixen (Universität Bayreuth). Er sah die Pro-

* Der Autor ist Rechtsanwalt in einem Fachverlag und Lehrbeauftragter für Medienrecht, Recht des World Wide Webs und Recht der neuen Medien an den Universitäten Bayreuth und Tübingen. 
motion als gemeinsames Projekt von Doktorand/in und Doktorvater bzw. -mutter. Bezüglich der inhaltlichen Kriterien einer rechtswissenschaftlichen Dissertation berief sich Rixen vor allem auf Schulze-Fielitz und dessen Reflektionen zur Wissenschaftlichkeit der Staatsrechtslehre.

Beide Referate lieferten zwei wichtige Leitmotive: die eigentliche - existentielle - Bedeutung von wissenschaftlichem Fragen (Repgen) und die ganz praktischen Überlegungen zur sinnvollen Konzeption von Promotionsvorhaben (Rixen).

\section{B. Blicke von und nach außen: Belgien, Großbritannien und die Schweiz}

Es ist gute Tradition der ZerF-Tagungen, ausgiebig rechtsvergleichende Vorträge zu bieten. Gerade für das diesjährige Thema waren die Blicke von und nach außen sehr wichtig. Sie zeigten, wie sich in der europäischen Wissenschaftslandschaft das interne Modell schon weitgehend etabliert hat.

Aus Belgien berichtete Mark van Hoecke (Universität Gent) über die „Konzeptionen von ,Rechtswissenschaft" und methodologische Bildung “. Wie schon die Überschrift seines Beitrags anklingen ließ, beschäftigte er sich ausschließlich mit den methodischen Aspekten des Promovierens. Für van Hoecke liegt dabei die Wissenschaftlichkeit von Promotionen ausschließlich in der angewandten Methodik. Dies begründete er mit den Anforderungen diverser Förderverfahren, in deren Antragsbegründungen die verwendeten Methoden eine zentrale Rolle spielten.

Mit dem Beitrag „Juristische Forschungsmethoden und die Promotion: die Situation in Großbritannien in vergleichender Perspektive" von Mathias Siems (Universität Durham, GB) blieb die Tagung zunächst auf der europäischen Ebene. Siems, der als Deutscher in Großbritannien einen Lehrstuhl an der Universität von Durham innehat, erlaubte einen Blick in das englische rechtswissenschaftliche Promotionssystem. Er verdeutlichte anschaulich, dass extern Promovierende als gut zahlende Gäste gerne gesehen sind. Sie werden nahezu umfassend betreut; bei Sprachschwierigkeiten wird Unterstützung angeboten.

Karsten Engsig Sørensen (Universität Aarhus) erläuterte „The Danish Setup for PhD in Law". Sehr pragmatisch hat sich die dänische Wissenschaft den Anforderungen angepasst: Es besteht ein dreispuriges System, bei dem zum „Dr. iur.“, zum „Ph.D“ und zum „Industrial Ph.D.“ promoviert werden kann. Letzteres ist ein Programm für Berufspraktiker und trägt deren Bedürfnissen besonders Rechnung.

Eine Bestandsaufnahme in Sachen rechtswissenschaftlicher Promotionen wäre wohl unvollständig, wenn nicht auch die Bologna-Perspektive eingenommen würde. Dies unternahm aus schweizerischer Sicht unter der vielsagenden Überschrift: „Zwischen Befürchtungen der Verschulung und Hoffnungen auf Qualitätssteigerung: Die Umsetzung von ,Bologna III“ in der Schweiz“ Michelle Cottier (Universität Basel). 


\section{Rechtsdidaktisches, Empirisches, Kritisches}

Nach den Blicken von und nach außen dachte Peter Tremp (Pädagogische Hochschule Zürich) über „Forschungsorientiertes Studium - Überlegungen aus bildungswissenschaftlicher Perspektive" nach. Tremp schlug vor, schon im Studium Forschung und damit wissenschaftliches Denken und Arbeiten stärker als didaktische Leitlinie zu integrieren. Daneben sei das Thema Forschung und Wissenschaft als eigener Themenkreis frühzeitig im Studium zu diskutieren und zu reflektieren.

Die überaus bedeutsamen Rahmenbedingungen des Promovierens wurden in einem Beitrag von Kai-Olaf Maiwald und Marc Torka (Universität Frankfurt am Main) aufgegriffen. Unter der Überschrift „Professionelle Promotionsbetreuung - jenseits von ,Meister' und ,Lehrling'? “ legten die beiden Vertreter des Instituts für Sozialforschung die bisherigen Ergebnisse ihres DFG-Projekts zur professionellen Promotionsbetreuung dar. Die beiden Referenten bestätigten auch aus soziologischer Sicht den Wandel von der individuellen zur strukturierten und institutionell formalisierten Promotionsbetreuung. Gleichzeitig räumten sie ein, dass entsprechende Untersuchungen zum Thema kaum existieren. Das noch bis 2016 laufende Projekt soll hier Abhilfe schaffen. Es werden dabei verschiedene Betreuungsformen empirisch untersucht.

Arne Pilniok und Hans-Heinrich Trute (Universität Hamburg) wiesen in ihrem Beitrag: „Externe Governance der Promotionsphase durch Forschungsförderorganisationen" sehr deutlich auf die große Steuerungsmacht der Forschungsförderorganisationen hin. Es sei, so die Referenten, schon lange nicht mehr die Rechtswissenschaft allein, die über Form und Inhalt der Doktorarbeiten bestimme. Durch die nähere Ausgestaltung ihrer Fördergeldbewilligungen werde ein erheblicher Einfluss ausgeübt, dem die Rechtswissenschaft relativ hilflos gegenüberstehe.

\section{Ertrag und Ausblick}

Die Tagung verdeutlichte eine nicht nur in der deutschen Rechtswissenschaft bestehende Tendenz, die Promotion als dritte Ausbildungsstufe zu etablieren. Die Zahl drei ist schön suggestiv und passt auch zu Bologna: Bachelor, Master, Doktor. Veränderte Rahmenbedingungen werden auf Dauer auch die Wahrnehmung und Bewertung der juristischen Doktorarbeiten und damit der Promotion als solcher verändern. Galt früher das Meister-Geselle-Paradigma, wie es Maiwald und Torka fast ein wenig karikierend schilderten - mit allen atmosphärischen Unwägbarkeiten -, so scheint sich auf breiter Front eine Institutionalisierung anzubahnen.

Maiwald und Torka haben schmunzelnd auf die Paradoxie hingewiesen, die nun dadurch entsteht, das man durch die Rundumbetreuung des Promovierenden zur wissenschaftlichen Selbstständigkeit (gar zum radikalen Fragen?) anleiten will. Die derzeitigen Formen und Inhalte juristischer Dissertationen, die ja den Hauptteil der Promotion ausmachen, sind ohnehin sehr vielfältig. Sie variieren ja schon von Rechtsgebiet zu Rechtsgebiet und dürfen und sollen von Lehrstuhl zu Lehrstuhl va- 
riieren. Es gibt wohl nicht die typische juristische Dissertation. Eine rechtshistorische Arbeit ist völlig unterschiedlich von einer rechtsdogmatischen oder rechtsvergleichenden Doktorarbeit. Was ihnen gemeinsam sein sollte, ist die Wissenschaftlichkeit.

Hier setzt behutsame Kritik des Verfassers an: Eigenartigerweise wurde der zentrale Begriff der „Wissenschaft“ und der „Wissenschaftlichkeit“ kaum thematisiert. Der wichtige Topos des radikalen Fragens, das wissenschaftliche Leitmotiv, das Repgen angeboten hatte, wurde von keinem Referat weiterbearbeitet.

Immerhin hat Tremp völlig zu Recht darauf hingewiesen, dass die Wissenschaftlichkeit wenn, dann frühzeitig eingeführt werden muss. Die Curricula der juristischen Fakultäten sind jedoch sehr weit von forschendem Lernen entfernt. Das hat gute und nachvollziehbare Gründe. Man gäbe den Studierenden Steine statt Brot, würde man mit ihnen wirklich Wissenschaft machen. Gelernt wird, was geprüft wird. In Klausuren der juristischen Staatsexamina werden keine rechtswissenschaftlichen Fragestellungen abgefragt. Es dominiert die praxisorientierte Falllösung; die eher wissenschaftlich orientierte Aufsatzform ist selten. Zu einer näheren Bestimmung dieses zentralen Begriffs kam es leider auch in der Diskussion nicht.

Im Gegenteil, es zeigte sich durchgängig allenfalls ein sehr blasser und auf die Methodik verengter Wissenschaftsbegriff. Dabei wäre eine durch ein wissenschaftstheoretisches Referat vorstrukturierte Diskussion aus Sicht des Verfassers sicher hilfreich gewesen. Es sei dabei eingeräumt, dass dies angesichts schon unterschiedlicher Wissenschaftskulturen in den drei großen Rechtsgebieten ein schwieriges Unterfangen gewesen wäre. Notwendig scheint es gleichwohl. Nicht nur die Behauptung von Wissenschaftlichkeit, sondern auch die konkrete Erläuterung für das jeweilige Rechtsfach wären wichtig, zum einen für die Doktoranden und Doktorandinnen selber, die nun deutlicher erkennen könnten, was von ihnen verlangt wird, zum anderen aber auch im Außenverhältnis. Angesichts der immer noch vorherrschenden Dominanz eines naturwissenschaftlich orientierten Wissenschaftsverständnisses ist über die Wissenschaftlichkeit von Rechtswissenschaft aufzuklären. 